\title{
A population-based validation study of the DCIS Score predicting recurrence risk in individuals treated by breast-conserving surgery alone
}

\author{
Eileen Rakovitch $^{1,2,3}$ - Sharon Nofech-Mozes ${ }^{3,4}$ - Wedad Hanna ${ }^{3,4}$. \\ Frederick L. Baehner ${ }^{5,6} \cdot$ Refik Saskin $^{2} \cdot$ Steven M. Butler $^{5}$ - Alan Tuck ${ }^{7}$. \\ Sandip Sengupta $^{8} \cdot$ Leela Elavathil $^{9}$ - Prashant A. Jani ${ }^{10,11} \cdot$ Michel Bonin ${ }^{12}$. \\ Martin C. Chang ${ }^{3,13}$ - Susan J. Robertson ${ }^{14}$ - Elzbieta Slodkowska ${ }^{4}$. \\ Cindy Fong ${ }^{2} \cdot$ Joseph M. Anderson $^{5}$ - Farid Jamshidian ${ }^{5}$. Dave P. Miller ${ }^{5}$. \\ Diana B. Cherbavaz ${ }^{5} \cdot$ Steven Shak $^{5} \cdot$ Lawrence Paszat $^{1,2,3}$
}

Received: 20 April 2015/Accepted: 8 June 2015/Published online: 29 June 2015

(c) The Author(s) 2015. This article is published with open access at Springerlink.com

\begin{abstract}
Validated biomarkers are needed to improve risk assessment and treatment decision-making for women with ductal carcinoma in situ (DCIS) of the breast. The Oncotype $\mathrm{DX}^{\circledR}$ DCIS Score (DS) was shown to predict the risk of local recurrence (LR) in individuals with low-risk DCIS treated by breast-conserving surgery (BCS) alone. Our objective was to confirm these results in a larger population-based cohort of individuals. We used an established population-based cohort of individuals diagnosed with DCIS treated with BCS alone from 1994 to 2003 with validation of treatment and outcomes. Central pathology assessment excluded cases with invasive cancer, DCIS $<2 \mathrm{~mm}$ or positive margins. Cox model was used to
\end{abstract}

Presented in part at the 37th Annual San Antonio Breast Cancer Symposium, December 9-13, 2014, San Antonio, Texas.

Electronic supplementary material The online version of this article (doi:10.1007/s10549-015-3464-6) contains supplementary material, which is available to authorized users.

\footnotetext{
Eileen Rakovitch

eileen.rakovitch@sunnybrook.ca

Department of Radiation Oncology, Sunnybrook Health Sciences Centre, Toronto, ON, Canada

2 Institute for Clinical Evaluative Sciences, Toronto, ON, Canada

3 University of Toronto, Toronto, ON, Canada

4 Department of Pathology, Sunnybrook Health Sciences Centre, Toronto, ON, Canada

5 Genomic Health, Inc., Redwood City, CA, USA

6 University of California, San Francisco (UCSF), San Francisco, CA, USA
}

determine the relationship between independent covariates, the DS (hazard ratio (HR)/50 $\mathrm{C}_{\mathrm{p}}$ units (U)) and LR. Tumor blocks were collected for 828 patients. Final evaluable population includes 718 cases, of whom 571 had negative margins. Median follow-up was 9.6 years. 100 cases developed LR following BCS alone (DCIS, $N=44$; invasive, $N=57$ ). In the primary pre-specified analysis, the DS was associated with any LR (DCIS or invasive) in ER+ patients (HR 2.26; $P<0.001)$ and in all patients regardless of ER status (HR 2.15; $P<0.001$ ). DCIS Score provided independent information on LR risk beyond clinical and pathologic variables including size, age, grade, necrosis, multifocality, and subtype (adjusted HR 1.68; $P=0.02$ ). DCIS was associated with invasive LR (HR 1.78; $P=0.04$ ) and DCIS LR (HR 2.43; $P=0.005$ ). The DCIS Score independently predicts and quantifies individualized recurrence risk in a population of patients with pure DCIS treated by BCS alone.

\footnotetext{
London Health Sciences Centre, London, ON, Canada

8 Kingston General Hospital, Kingston, ON, Canada

9 Henderson General Hospital, Hamilton, ON, Canada

10 Thunder Bay Regional Health Sciences Centre, Thunder Bay, ON, Canada

11 Northern Ontario School of Medicine, Thunder Bay, ON, Canada

12 Health Sciences North Sudbury, Sudbury, ON, Canada

13 Mount Sinai Hospital, Toronto, ON, Canada

14 The Ottawa Hospital, Ottawa, ON, Canada
} 
Keywords Ductal carcinoma in situ - DCIS treatment . DCIS Score - Gene expression profiling - 12-Gene assay . Local recurrence

\section{Introduction}

Ductal carcinoma in situ (DCIS) is a non-invasive form of breast cancer that comprises up to $25 \%$ of mammographically detected breast cancers [15]. The goals of treatment are to minimize the risk of local recurrence (LR) and invasive breast cancer while maximizing breast preservation. Most women with newly diagnosed DCIS will be treated with breast-conserving surgery (BCS) with or without radiation treatment (RT). Randomized trials have demonstrated that adding RT after surgical excision reduces the relative risk of LR by $\sim 50 \%[7,8$, $11,14,16,17,19,38,42]$. However, only one-half of DCIS patients treated by BCS receive RT, perhaps reflecting clinicians' assumption that women at low risk of recurrence following treatment by BCS alone can be accurately identified $[6,20,22]$. To date, reproducible and reliable methods using clinico-pathologic features to identify patients at low risk of LR following BCS alone have not been established and there is a need to improve individualized treatment decisions to minimize both under and over treatment $[1,9,21,35,37,45,46]$. The 2009 National Institutes of Health State-of-the-Science Conference included the recommendation that future research on DCIS focus on the development and validation of risk stratification models to optimize treatment recommendations for each individual diagnosed with DCIS [1].

The Oncotype DX ${ }^{\circledR}$ DCIS Score is a multigene expression assay for DCIS patients that generates individualized estimates of 10-year risk of any LR (DCIS or invasive) and invasive LR following treatment by BCS alone [39]. The DCIS Score is generated from an algorithm that includes 12 (seven cancer related and five reference genes) of the 21 genes in the Recurrence Score assay [30]. The continuous DCIS Score was shown to predict an individual's risk of LR in the Eastern Cooperative Oncology Group (ECOG) E5194 prospective cohort study of low-risk DCIS treated by BCS alone [39]. However, participants in ECOG 5194 were highly selected for having DCIS with an expected low risk of LR. Additional data from a diverse population of women with DCIS treated by BCS alone is desired to confirm the prognostic ability of the DCIS Score. The objective of this study is to evaluate if the DCIS Score is an independent predictor of LR in a population of individuals with DCIS treated with BCS alone.

\section{Methods}

\section{Ontario DCIS cohort}

The methods used to establish the Ontario populationbased DCIS cohort have been previously described [32]. The study population includes 5752 women diagnosed with DCIS. Cases treated by mastectomy $(N=1785)$ or diagnosed with invasive breast cancer within 6 months of DCIS diagnosis $(N=172)$ were excluded. There were 3795 cases with DCIS treated by BCS (alone or with RT). 628 cases were excluded following pathology review (494 had invasive cancer or microinvasion, 100 had benign diagnosis and 34 had LCIS). We excluded six cases with bilateral DCIS, eight cases with prior mastectomy and one case who died within 6 months of DCIS diagnosis. 182 cases initially diagnosed as LCIS were found to have DCIS following pathology review of cases with LCIS and were added to the DCIS cohort. The population cohort includes 3320 cases with pure DCIS; 1658 treated by BCS alone and 1662 by BCS + RT.

\section{Pathology}

We performed a centralized pathology review in 2720 cases of the population cohort by an expert breast pathologist. Pathology review was performed on original $\mathrm{H} \& \mathrm{E}$ slides, using recuts when available and core biopsies in cases with no residual disease on excision. If slides were not available, the original report was abstracted. Nuclear grade (low, intermediate, high, unreported), comedo necrosis (present/absent), multifocality (present/absent), tumor size (mm/unreported), and margin status (positive, negative, unreported) was assessed [27]. Margin status was defined as "positive" if there were tumor cells identified at the inked resection margin. Multifocal lesions were defined as having more than one distinct focus of DCIS with at least $5.0 \mathrm{~mm}$ of intervening benign tissue, confined to a single quadrant of the breast [36]. Tumor size and margin width could not be assessed without slides for all the blocks or where the gross description was incomplete.

\section{Treatment}

To obtain data on treatment and outcomes, deterministic linkage was performed with the Canadian Institute for Health Information (CIHI) database of hospital discharge summaries, the Ontario Health Insurance Plan (OHIP) database of physician billings, the Registered Persons Database (RPDB) and the Ontario Cancer Registry (OCR) database [33]. For each case, we identified all breast surgical procedures with validation by chart review or 
pathology review. The date of diagnosis is the date of the initial breast surgery associated with the DCIS diagnosis. Tamoxifen usage in women $\geq 65$ years was identified in the Ontario Drug Benefit database. Tamoxifen usage in women $<65$ years of age was not available.

\section{Outcomes}

Outcomes were determined from the date of diagnosis of DCIS. We identified breast surgical procedures performed more than 6 months after diagnosis, linked with the OCR and CIHI databases and reviewed available pathology reports to determine recurrence laterality and histology. LR is defined as invasive breast cancer or DCIS in the ipsilateral breast 6 months after DCIS diagnosis. To include individuals with invasive LR after an initial DCIS LR, no censoring at time of initial DCIS LR was performed. Contralateral breast cancer is defined by the presence of DCIS or invasive breast cancer in the opposite breast. The date of death was determined from the RPDB. The last date of follow-up is March 31, 2010.

\section{Gene assay}

Cases with DCIS lesions $<2 \mathrm{~mm}$ were excluded and nonDCIS elements were manually micro-dissected to enrich DCIS [39]. RNA was extracted from $30 \mu \mathrm{m}$ sections if DCIS measured $\geq 5.5 \mathrm{~mm}$, or from $60 \mu \mathrm{m}$ micro-dissected sections if DCIS measured $<5.5 \mathrm{~mm}$. The Oncotype $\mathrm{DX}^{\circledR}$ Breast Cancer Assay was performed as previously described $[12,13,30]$. TaqMan ${ }^{\circledR}$ PCR reactions were conducted in 384-well micro titer plates on Roche LightCycler $^{\circledR} 480$ (Roche Diagnostics, Indianapolis, IN), and gene expression was quantified by the second derivative maximum, Crossing point, $(\mathrm{Cp})$ method, in accordance with manufacturers instruction.

The DCIS Score (12 genes) is scaled as a continuous variable from 0 to 100 , and is derived from the reference normalized gene expression measurements in four prespecified steps, as reported previously [39]. Three risk categories used in prior studies were pre-specified for this study: (1) low-risk (DCIS Score <39); (2) intermediaterisk (DCIS Score 39-54); and (3) high-risk (DCIS Score $\geq 55$ ).

\section{Statistical analysis and study endpoints}

Study methods, DCIS Score algorithm, and statistical analysis plan were finalized before the study was conducted [39]. The primary objective was to determine whether the DCIS Score is associated with the risk of any LR (DCIS or invasive) in patients treated with BCS alone with negative margins. A secondary analysis included patients with positive/unknown margins. The primary analysis was conducted in two stages: (1) restricted to cases with estrogen receptor (ER)-positive DCIS as determined by RT-PCR and (2) for all patients regardless of ER status. Conditional fixed sequential (hierarchical) hypothesis testing was utilized to preserve the overall family wise type I error rate for the primary analysis at the 0.05 level [44]. The association was tested in each case using Cox proportional hazards models, and statistical significance was based on a likelihood ratio test with $P \leq 0.05$. Secondary analyses examined the association of the DS with invasive LR and with DCIS LR. For the secondary analyses of DCIS LR, patients were censored at the time of invasive LR, and for the analyses of invasive LR, patients continued to be followed for invasive LR following a DCIS LR. A limited number of clinico-pathologic variables were prespecified for subgroup analyses and potential covariate adjustment. To assess the independent association of the DCIS Score with LR with adjustment for other clinicopathologic characteristics using multivariable Cox models with a $P$ value cutoff of 0.05 . Spearman correlations were computed to assess the association between DCIS Score and clinico-pathologic characteristics. Diagnostics based on Martingale and Schoenfeld residuals supported the pre-specified assumptions of linearity and proportional hazards [41].

\section{Results}

\section{Patient characteristics}

The population cohort includes 3320 individuals with pure DCIS (Fig. 1). There were 1658 cases treated by BCS alone ( $N=1061$ with negative margins). Median followup was 9.6 years. We obtained tissue blocks for 828 patients treated with BCS alone $50 \%$ of provincial cohort) and for each case an optimal representative tissue block was sent for gene analysis. 110 cases were excluded: 10 with no evidence of DCIS, four with evidence of invasive carcinoma on further analysis, 68 cases with insufficient RNA and 28 cases with poor qPCR sample quality.

The final evaluable study cohort includes 718 cases treated by BCS alone; of these 571 cases had negative margins (Table 1). The median age at diagnosis was 61 years. There were $100 \mathrm{LR}$ events $[N=44$ DCIS, $N=57$ invasive (one case developed invasive LR after DCIS LR)]. The overall 10-year rate of LR was $19.2 \%$. A comparison of characteristics of individuals included in the study cohort to those not included is listed in Supplementary Table 1. 
Fig. 1 Consolidated standards of reporting trials flow diagram for study numbers. DCIS ductal carcinoma in situ, GHI Genomic Health, Inc., $B C S$ breastconserving surgery

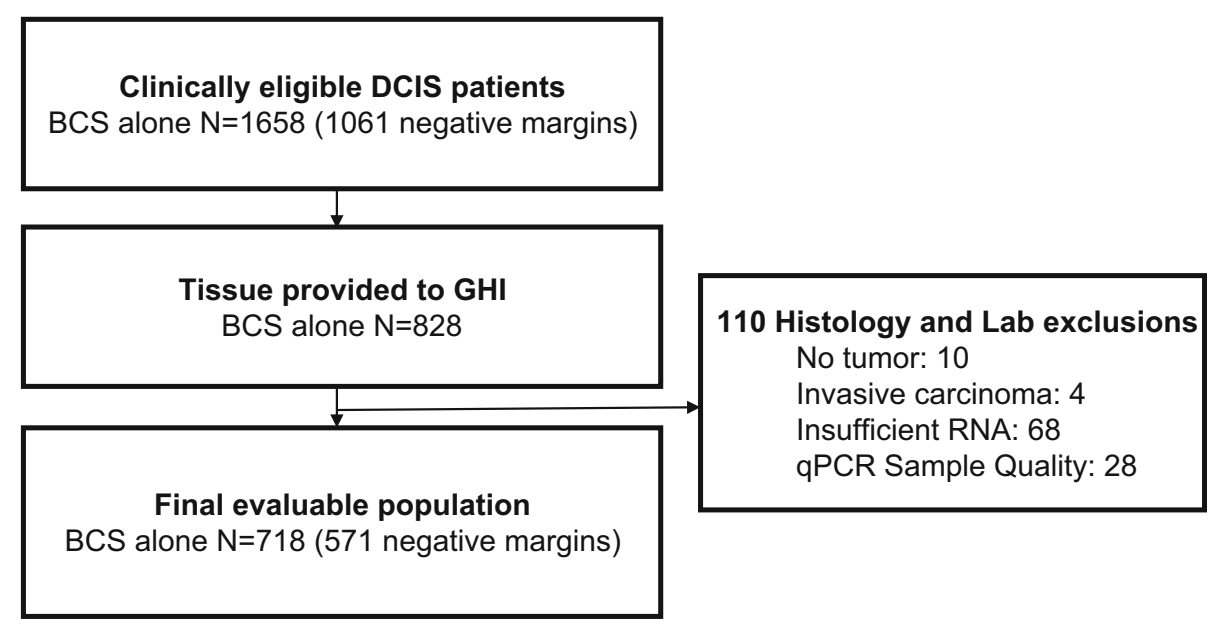

\section{Predictors of local recurrence}

In the pre-specified primary analysis, the DCIS Score was significantly associated with the risk of LR in patients with ER-positive DCIS treated with BCS alone with negative margins (hazard ratio [HR] 2.26; $95 \%$ CI 1.41, 3.59; $P<0.001 ;$ Table 2). The DCIS Score was also associated with LR in all patients treated by BCS alone with negative margins irrespective of ER status (HR 2.15; $95 \%$ CI 1.43, 3.22; $P<0.001$; Table 2). Since $94.7 \%$ of patients treated with BCS alone had ER-positive DCIS (by RT-PCR), data for all cases regardless of ER status is presented (Fig. 2, panels a, b). The DCIS Score was also significantly associated with invasive LR (HR 1.78; $95 \%$ CI 1.03, 3.05; $P=0.04$ ) and DCIS LR (HR 2.43, $95 \%$ CI 1.31, 4.42; $P=0.005$ ) (Table 2). On univariable analysis, other factors associated with the development of LR include the presence of multifocality, tumor size, subtype, nuclear grade, and comedo necrosis (Supplemental Table 2).

On multivariable analysis significant predictors of LR include the DCIS Score (HR 1.68; $95 \%$ CI 1.08, 2.62), the presence of multifocality (HR 1.97; $95 \%$ CI 1.27, 3.02), tumor size $>10 \mathrm{~mm}$ (HR 2.07; $95 \%$ CI 1.15, 3.83), age at diagnosis (HR 1.75; $95 \%$ CI 1.07, 2.76), and architectural subtype (HR 1.63 solid vs. cribriform; $95 \%$ CI 0.97, 2.88). Comedo necrosis and grade were not independent predictors of LR (Table 3). As a secondary analysis without restrictions on margin status $(N=718)$, there were 147 patients with positive/unknown margins. Adjusting for margin status, the HR for DCIS Score was 2.11 (95\% CI $1.43,3.09 ; P<0.001)$.

\section{Local recurrence by DCIS Score risk group}

Two-thirds $(62.2 \%)$ of women in the study cohort had a low risk score, $16.6 \%$ had an intermediate risk score and
$21.2 \%$ had a high risk score. The 10-year rates of LR were $12.7,33.0$, and $27.8 \%$, respectively (log rank $P<0.001$ ) (Fig. 2, panel a). The corresponding 10-year rates of invasive LR were 8.0, 20.9 and $15.5 \%(P=0.03)$ (Supplemental Fig. 1, panel a) and DCIS LR were 5.4, 14.1 and $13.7 \%$ for DCIS LR for the low, intermediate and high DCIS Score groups, respectively $(P=0.002)$ (Supplemental Fig. 1, panel b).

We refit the multivariable model using a dichotomous indicator for the DCIS risk groups. The hazard ratio for cases in the intermediate/high DCIS Score group compared to those in the low-risk group was 1.88 (95\% CI 1.24, $2.87 ; P=0.003)$. The 10 -year rate of contralateral breast cancer in the BCS alone group with negative margins was $4.8 \%$. There was no difference in the rates of contralateral breast cancer (DCIS or invasive) according to risk group.

\section{Subgroup analyses}

We evaluated the rates of LR by age at diagnosis and the presence of baseline pathological features of DCIS. With the exception of the presence of multifocality, individuals in the low DCIS Score group had lower 10-year rates of LR than those in the intermediate or high score groups (Fig. 3). Excluding individuals with multifocal DCIS, the 10-year rates of LR for the low, intermediate and high DCIS Score groups were 9.7, 27.1, and $27.0 \%(\log$ rank $P<0.001)$ (Supplemental Fig. 2, panel a); the corresponding 10-year rates of invasive LR were 5.6, 16.7, and $16.3 \%$ $(P=0.02)$; and, the 10 -year rates of DCIS LR were 4.3 , 11.4 , and $12.1 \%$, respectively $(P=0.02)$. (Supplemental Fig. 2, panels b, c).

The DCIS Scores were weakly correlated with age at diagnosis and pathological features of DCIS (correlation coefficients ranged from ( -0.03 to 0.47$)$ (Supplementary Fig. 4, panels a-f). 
Table 1 Patient and tumor characteristics for patients with pure DCIS treated by breast-conserving surgery alone with negative resection margins

\begin{tabular}{|c|c|}
\hline Characteristic & BCS alone $(N=571)$ \\
\hline Age (median years) & 61 \\
\hline \multicolumn{2}{|l|}{ Age category ${ }^{\mathrm{a}}$} \\
\hline$<50$ years & $110(19.3 \%)$ \\
\hline$\geq 50$ years & $459(80.7 \%)$ \\
\hline \multicolumn{2}{|l|}{ Multifocality } \\
\hline Absent/not reported & $457(80.0 \%)$ \\
\hline Present & $114(20.0 \%)$ \\
\hline \multicolumn{2}{|l|}{ Nuclear grade } \\
\hline Low & $55(9.6 \%)$ \\
\hline Moderate & $332(58.1 \%)$ \\
\hline High & $184(32.2 \%)$ \\
\hline \multicolumn{2}{|l|}{ Comedo necrosis } \\
\hline Absent & $221(38.7 \%)$ \\
\hline Present & $350(61.3 \%)$ \\
\hline \multicolumn{2}{|l|}{ Tumor size category } \\
\hline Missing & $281(49.2 \%)$ \\
\hline$>10 \mathrm{~mm}$ & $140(24.5 \%)$ \\
\hline$\leq 10 \mathrm{~mm}$ & $150(26.3 \%)$ \\
\hline \multicolumn{2}{|l|}{ Subtype } \\
\hline Solid & $358(62.7 \%)$ \\
\hline Cribriform & $175(30.6 \%)$ \\
\hline Micropapillary & $11(1.9 \%)$ \\
\hline Other & $27(4.7 \%)$ \\
\hline \multicolumn{2}{|l|}{ DCIS Score group } \\
\hline Low & $355(62.2 \%)$ \\
\hline Intermediate & $95(16.6 \%)$ \\
\hline High & $121(21.2 \%)$ \\
\hline \multicolumn{2}{|l|}{ ER status } \\
\hline Negative & $30(5.3 \%)$ \\
\hline Positive & $541(94.7 \%)$ \\
\hline \multicolumn{2}{|l|}{ HER2 Status } \\
\hline Negative & $420(73.6 \%)$ \\
\hline Equivocal & $51(8.9 \%)$ \\
\hline Positive & $100(17.5 \%)$ \\
\hline
\end{tabular}

${ }^{a}$ Two patients were missing age

\section{Discussion}

This study validates that the DCIS Score is significantly associated with the risk of LR (DCIS or invasive) in a population of patients diagnosed with pure DCIS treated with BCS alone with negative margins. We also found the DCIS Score is associated with the risk of invasive LR and DCIS LR and provides independent information on the risk of LR in individuals selected for treatment by BCS alone.
Table 2 Association of the DCIS Score and the development of local recurrence in patients treated by breast-conserving surgery alone with negative resection margins: univariable analysis

\begin{tabular}{lcc}
\hline Endpoint & $\mathrm{HR} / 50 \mathrm{U}(95 \% \mathrm{CI})^{*}$ & $P$ value* \\
\hline Local recurrence in ER+ DCIS & $2.26(1.41,3.59)$ & $<0.001^{\S}$ \\
In all patients regardless of ER status & \\
Local recurrence & $2.15(1.43,3.22)$ & $<0.001^{\S}$ \\
Invasive local recurrence & $1.78(1.03,3.05)$ & 0.04 \\
DCIS local recurrence & $2.43(1.31,4.42)$ & 0.005 \\
\hline
\end{tabular}

* Profile likelihood CI, likelihood ratio $P$ value

$\S$ The primary analysis in patients treated with BCS alone with negative margins was conducted hierarchically in two stages: stage 1 in ER+ patients and stage 2 in all patients regardless of ER status

Most women diagnosed with DCIS will be candidates for BCS. The decision to recommend additional treatment such as radiation relies on estimates of the baseline risk of LR following treatment by BCS alone. For individuals estimated to have low LR risk, guidelines include the option for treatment by BCS alone; however, the ability to identify individuals at low risk of recurrence after treatment by BCS has been inconsistent. Current clinicopathologic criteria do not reliably identify individuals with a low risk of LR [29]. As a result, studies report that $30-50 \%$ of individuals with DCIS are treated by BCS alone including some individuals with higher risk DCIS who do not meet the eligibility criteria of the ECOG 5194 or RTOG 9804 clinical trials for low-risk DCIS [6, 22, 23, 32, 43]. In E5194 the 10-year LR rate among cases with low- or intermediate-grade DCIS and tumor size $\leq 2.5 \mathrm{~cm}$ was 14.6 and $19.0 \%$ for those with small $(\leq 1 \mathrm{~cm})$ highgrade DCIS [39]. Our population-based cohort includes individuals with confirmed pure DCIS selected for treatment by BCS alone and despite having lower risk features compared to those treated with RT, the 10-year risk of LR was $19.2 \%$ demonstrating that clinico-pathologic criteria alone are insufficient to reliably identify individuals with a low risk of recurrence [32].

Individuals in our population cohort were not as highly selected as those in the ECOG E5194 study; for example, $32 \%$ had high-grade DCIS, $45 \%$ had margin width between 1 and $3 \mathrm{~mm}$. Despite these differences, the risks of LR in each pre-specified DCIS risk group were remarkably similar. The 10-year rates of LR for cases with low, intermediate or high scores were $12.7,33.0$, and $27.8 \%$ compared to 10.6, 26.7 and $25.9 \%$ reported in the E5194 analysis, respectively. These findings validate the DCIS Score as a predictor of LR in a more diverse, populationbased cohort compared to participants of the E5194 study.

The DCIS Score was not strongly correlated with age at diagnosis, or pathologic features of DCIS. On multivariable 


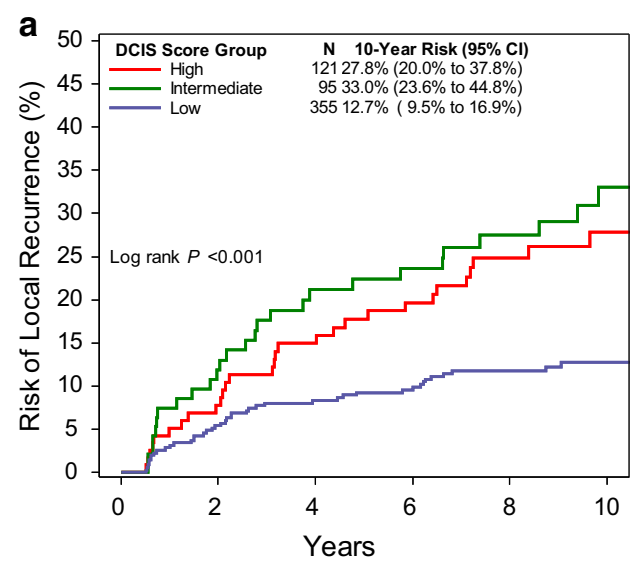

Number at risk

$\begin{array}{rcccccc}\text { High } & 121 & 104 & 92 & 83 & 61 & 42 \\ \text { Intermediate } & 95 & 77 & 66 & 63 & 48 & 30 \\ \text { Low } & 355 & 329 & 304 & 289 & 217 & 143\end{array}$

Fig. 2 Kaplan-Meier estimates of the 10-year risk of local recurrence by DCIS Score Group (a) and Cox model estimates of 10-year local recurrence risk according to the continuous DCIS Score (b), in patients treated with BCS alone and negative margins. The number of patients at risk is included for each pre-specified risk group based on

Table 3 Predictors of local recurrence in patients with DCIS treated by breast-conserving surgery alone with negative resection margins: multivariable analysis

\begin{tabular}{llll}
\hline Characteristic & $N$ & HR $(95 \% \mathrm{CI})^{*}$ & $P$ value* \\
\hline $\begin{array}{l}\text { DCIS Score (HR/50 U) } \\
\text { Multifocality }\end{array}$ & 571 & $1.68(1.08,2.62)$ & 0.02 \\
$\quad$ Absent/unknown & 457 & 1.0 & 0.003 \\
$\quad$ Present & 114 & $1.97(1.27,3.02)$ & \\
Tumor size & & & $0.01^{\S}$ \\
$\quad \leq 10 \mathrm{~mm}$ & 150 & 1.0 & \\
$>10 \mathrm{~mm}$ & 140 & $2.07(1.15,3.83)$ & \\
Age & & & 0.03 \\
$\quad \geq 50$ & 459 & 1.0 & \\
$\quad<50$ & 110 & $1.75(1.07,2.76)$ & \\
DCIS tumor subtype & & & \\
$\quad$ Cribriform & 175 & 1.0 & \\
$\quad$ Solid & 358 & $1.63(0.97,2.88)$ & \\
Other & 38 & $2.75(1.17,6.04)$ & \\
\hline
\end{tabular}

* Profile likelihood CI, likelihood ratio $P$ value

$\S \mathrm{P}$ value is for tumor size in cases where it is available

* Missing values included as an indicator variable (not shown)

analysis, other factors associated with the development of LR include the presence of multifocality, tumor size, age $<50$ years at diagnosis and subtype. Nuclear grade and comedo necrosis were not independent predictors of LR on multivariable analysis after adjustment for other characteristics. The significance of nuclear grade as a predictor of

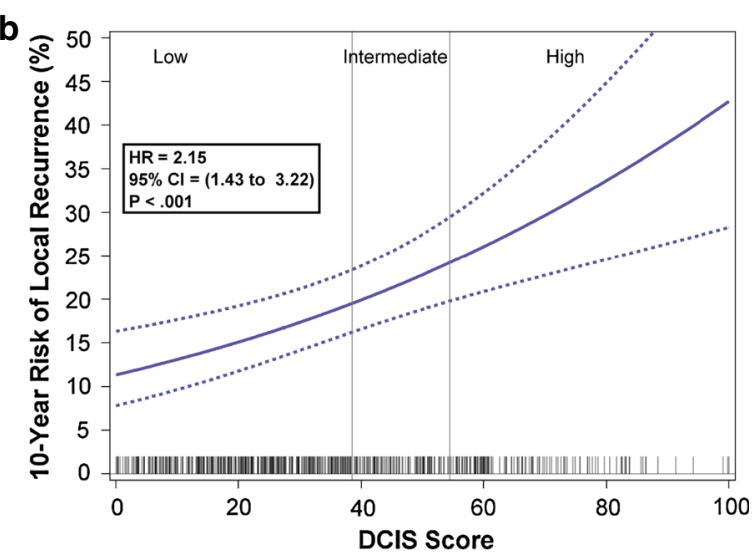

the DCIS Score of low $(<39)$, intermediate (39-54) and high $(>55)$. The risk based on continuous DCIS Score assumes a monotone incremental risk as DCIS Score increases. Although formal statistical tests for non-linearity were negative, the Kaplan-Meier estimates suggest that a non-linear effect is plausible

LR has not been consistently identified in past studies [10, 26, 39]. This may be related to the correlation of grade with other pathological features, variability of grading systems, inter-observer variability, or heterogeneity of grade within a DCIS lesion [4, 18, 28, 39].

There was consensus among the expert breast pathologists involved in this study to a priori define the presence of multifocal DCIS as the presence of at least two foci of DCIS separated by at least $5 \mathrm{~mm}$ [36]. In a previous analysis based on an institutional cohort, we found that the presence of multifocal DCIS was an independent predictor of LR [34]. We used the same definition in order to evaluate the impact of multifocality on the risk of LR in the population cohort. Using the predefined definition, $20 \%$ of patients in the population cohort had multifocal DCIS. The 10-year risk of LR was $33.6 \%$ for those with multifocality compared to $15.5 \%$ for those without multifocal disease (adjusted HR 1.97; $95 \%$ CI 1.27, 3.02; $P=0.003$ ). The reasons why individuals with multifocal DCIS experienced a higher risk of LR are unclear. It is possible that the presence of multifocality is associated with a greater burden of residual disease or it may be a marker of molecular heterogeneity [3]. Among individuals without multifocality, the 10-year risk of LR among individuals with a low DCIS score was $9.7 \%$ compared to 27.1 and $27.0 \%$ for those with intermediate and high risk scores, respectively.

There are noteworthy clinical implications regarding our findings. Currently, the eligibility criteria for the E5194 and RTOG 9804 studies is used by clinicians to identify individuals with an expected low risk of LR following BCS 
Fig. 3 Subgroup analyses of the 10-year LR risk by DCIS Score Group. The left side of the figure show the Kaplan-Meier estimates of the 10-year risk of any local recurrence (with $95 \%$ CI) according to the DCIS Score pres-pecified risk groups. Blue boxes are estimates for the low DCIS Score risk group and are generally to the left of the overall LR rate of $19.2 \%$. Green boxes are estimates for the intermediate DCIS Score risk group. Red boxes are estimate for the high DCIS Score risk group and are generally to the right of the overall LR risk estimate. The box size is proportional to the number of patients. The right side of the figure shows the hazard ratios for LR risk, with $95 \%$ CIs. The hazard ratios are calculated for a 50-point difference in the continuous DCIS Score

\section{Subgroup DCIS Group}

\section{All Patients}

Low risk

Intermediate risk

High risk

Age $<50$

All Patients

Low risk

Intermediate risk

High risk

Age $\geq \mathbf{5 0}$

All Patients

Low risk

Intermediate risk

High risk

Multifocality: absent

All Patients

Low risk

Intermediate risk

High risk

Multifocality: present

All Patients

Low risk

Intermediate risk

High risk

Subtype: solid

All Patients

Low risk

Intermediate risk

High risk

Subtype: cribriform

All Patients

Low risk

Intermediate risk

High risk

Low/int. grade

All Patients

Low risk

Intermediate risk

High risk

High grade

All Patients

Low risk

Intermediate risk

High risk

Comedonecrosis: absent

\section{All Patients}

Low risk

Intermediate risk

High risk

Comedonecrosis: present

\section{All Patients}

Low risk

Intermediate risk

High risk
No. of

Patients

571
355
95
121

121

110

71
18

21

459

282

77
100

457

298

72
87

114

57
23

34

358

185

70
103

103

175

141
21

21
13

387

291
60

60
36

184

64
35
85

85

221
185
22
14

14

350

170

73
107
19.2

20
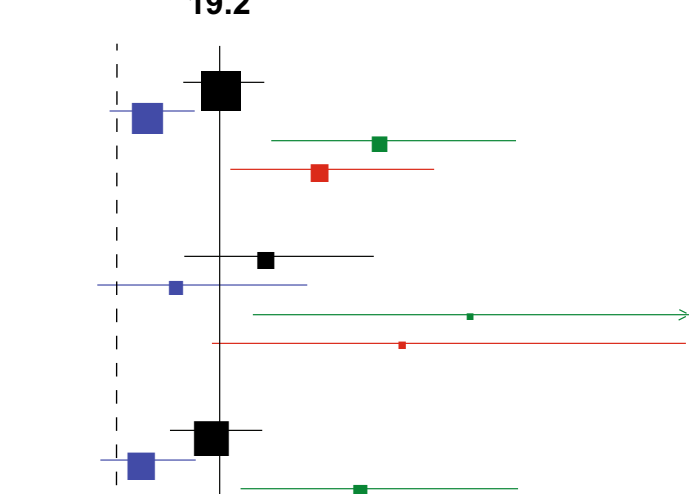
the DCIS Score provides individualized estimates of recurrence risk. This can help clinicians and patients better weigh recurrence risks against the potential benefits and toxicities of treatment.

The present study has several notable strengths. The study cohort is population-based and includes a more diverse population of individuals with DCIS selected for treatment by BCS alone. We performed an extensive, systematic pathologic review by expert breast pathologists using contemporary classifications to confirm the diagnosis of pure DCIS and a rigorous, predefined statistical analytical plan, including pre-specified cut-points for the DCIS Score and study objectives. We applied the same prespecified cut-points for the DCIS Score used in the E5194 analysis and found that the DCIS Score is valid in a population of women with DCIS. The low-risk group appears to have a lower risk of LR than those in the intermediate or high-risk group (HR 0.53, $95 \%$ CI 0.34, 0.80) (the study was not powered to evaluate difference between the intermediate and high-risk group). In addition, for the lowrisk group, we did not observe a lower risk of LR with lower cut-points. We did observe a significant decrease in the rate of LR over time. However, for each time period, individuals in the low-risk DCIS Score group had a significantly lower risk of LR than those in the intermediate or high-risk groups. There was no interaction between the DCIS Score and year of diagnosis and the DCIS Score remained significantly associated with the risk of LR on multivariable analysis adjusting for year of diagnosis. The 10-year risk LR among individuals treated in year 2000-2003, with median follow-up interval was 7.9 years, was $12.1 \%$; for those in the low-risk DCIS Score group treated during this time period without multifocality, the 10-year risk of LR was $5.8 \%(2.9-11.3 \%)$ compared to 20.7 and $22.4 \%,(P=0.03)$ for the intermediate and highrisk DCIS Score groups.

The study has several potential limitations. Patients were not randomized and were selected for treatment by BCS alone based on clinico-pathologic features and patient preference. During the time interval of this study, many pathology reports lacked tumor size and resection margin width information [31, 40]. Therefore, margin width and tumor size data were incomplete. In addition, data on clinical presentation or family history of breast cancer which may predict for LR were not available [24]. Tamoxifen utilization during the time period of this study was limited. Complete data on tamoxifen usage (in younger women) was not available. Among women older than 65 years, only $17 \%$ received tamoxifen and compliance was not available. However, $95 \%$ of cases treated with BCS alone had ER-positive DCIS and therefore slightly lower event rates might be expected with tamoxifen administration [2, 5, 25].
In summary, we confirm that the DCIS Score independently predicts the risk of LR in a population of individuals with DCIS who were treated with BCS. The DCIS Score quantifies individualized risk of LR which can help guide treatment recommendations and help reduce over treatment for women at low risk and under treatment for those with a significant risk of recurrence who may benefit from further treatment.

Acknowledgments This work was supported in part by funding from the Canadian Cancer Society Research Institute and by the Institute for Clinical Evaluative Sciences (ICES), which is funded by an annual grant from the Ontario Ministry of Health and Long-Term Care (MOHLTC). The opinions, results and conclusions reported in this paper are those of the authors and are independent from the funding sources. No endorsement by ICES or the Ontario MOHLTC is intended or should be inferred. Genomic Health, Inc. provided funding to the Sunnybrook Research Institution in support of this work. Dr. Rakovitch is the LC Campbell Chair for Breast Cancer Research.

Conflict of interest This work was supported in part by funding from the Canadian Cancer Society Research Institute. Genomic Health, Inc. provided funding to the Sunnybrook Research Institution in support of this work. Dr. Baehner, Dr. Butler, Mr. Miller, Dr. Charbavaz, Dr. Anderson, and Dr. Jamshidian are employees of Genomic Health, Inc. and they own stock in Genomic Health, Inc. Dr. Shak is an executive level employee at Genomic Health, Inc. and owns stock in Genomic Health, Inc.

Open Access This article is distributed under the terms of the Creative Commons Attribution-NonCommercial 4.0 International License (http://creativecommons.org/licenses/by-nc/4.0/), which permits any noncommercial use, distribution, and reproduction in any medium, provided you give appropriate credit to the original author(s) and the source, provide a link to the Creative Commons license, and indicate if changes were made.

\section{References}

1. Allegra CJ, Aberle DR, Ganschow P, Hahn SM, Lee CN, MillonUnderwood S, Pike MC, Reed SD, Saftlas AF, Scarvalone SA, Schwartz AM, Slomski C, Yothers G, Zon R (2010) State-of-thescience conference: diagnosis and management of ductal carcinoma in situ (DCIS). J Natl Cancer Inst 102:161-169

2. Allred DC, Anderson SJ, Paik S, Wickerham DL, Nagtegaal ID, Swain SM, Mamounas EP, Julian TB, Geyer CE Jr, Costantino JP, Land SR, Wolmark N (2012) Adjuvant tamoxifen reduces subsequent breast cancer in women with estrogen receptor-positive ductal carcinoma in situ: a study based on NSABP protocol B-24. J Clin Oncol 30:1268-1273. doi:10.1200/JCO.2010.34. 0141

3. Allred DC, Wu Y, Mao S, Nagtegaal ID, Lee S, Perou CM, Mohsin SK, O'Connell P, Tsimelzon A, Medina D (2008) Ductal carcinoma in situ and the emergence of diversity during breast cancer evolution. Clin Cancer Res 14:370-378. doi:10.3390/ e14020370

4. Badve S, Gray RJ, Baehner FL, Solin LJ et al. (2012) Correlation between the DCIS Score and traditional clinicopathologic features in the prospectively designed E5194 clinical validation study. J Clin Oncol 30 
5. Badve SS, Baehner FL, Gray RP, Childs BH, Maddala T, Liu ML, Rowley SC, Shak S, Perez EA, Shulman LJ, Martino S, Davidson NE, Sledge GW, Goldstein LJ, Sparano JA (2008) Estrogen- and progesterone-receptor status in ECOG 2197: comparison of immunohistochemistry by local and central laboratories and quantitative reverse transcription polymerase chain reaction by central laboratory. J Clin Oncol 26:2473-2481. doi:10.1200/JCO.2007.13.6424

6. Baxter NN, Virnig BA, Durham SB, Tuttle TM (2004) Trends in the treatment of ductal carcinoma in situ of the breast. J Natl Cancer Inst 96:443-448

7. Bijker N, Meijnen P, Peterse JL, Bogaerts J, Van Hoorebeeck I, Julien JP, Gennaro M, Rouanet P, Avril A, Fentiman IS, Bartelink H, Rutgers EJ (2006) Breast-conserving treatment with or without radiotherapy in ductal carcinoma-in situ: ten-year results of European Organisation for Research and Treatment of Cancer randomized phase III trial 10853-a study by the EORTC Breast Cancer Cooperative Group and EORTC Radiotherapy Group. J Clin Oncol 24:3381-3387. doi:10.1200/JCO.2006.06.1366

8. Bijker N, Peterse JL, Duchateau L, Julien JP, Fentiman IS, Duval C, Di Palma S, Simony-Lafontaine J, de Mascarel I, van de Vijver MJ (2001) Risk factors for recurrence and metastasis after breast-conserving therapy for ductal carcinoma-in situ: analysis of European Organization for Research and Treatment of Cancer Trial 10853. J Clin Oncol 19:2263-2271

9. Burstein HJ, Polyak K, Wong JS, Lester SC, Kaelin CM (2004) Ductal carcinoma in situ of the breast. $\mathrm{N}$ Engl J Med 350:1430-1441. doi:10.1056/NEJMra031301350/14/1430

10. Collins LC, Achacoso N, Nekhlyudov L, Fletcher SW, Haque R, Quesenberry CP Jr, Puligandla B, Alshak NS, Goldstein LC, Gown AM, Schnitt SJ, Habel LA (2009) Relationship between clinical and pathologic features of ductal carcinoma in situ and patient age: an analysis of 657 patients. Am J Surg Pathol 33:1802-1808

11. Correa C, McGale P, Taylor C, Wang Y, Clarke M, Davies C, Peto R, Bijker N, Solin L, Darby S (2010) Overview of the randomized trials of radiotherapy in ductal carcinoma in situ of the breast. J Natl Cancer Inst Monogr 2010:162-177. doi:10. 1093/jncimonographs/lgq039

12. Cronin M, Pho M, Dutta D, Stephans JC, Shak S, Kiefer MC, Esteban JM, Baker JB (2004) Measurement of gene expression in archival paraffin-embedded tissues: development and performance of a 92-gene reverse transcriptase-polymerase chain reaction assay. Am J Pathol 164:35-42

13. Cronin M, Sangli C, Liu ML, Pho M, Dutta D, Nguyen A, Jeong J, Wu J, Langone KC, Watson D (2007) Analytical validation of the Oncotype DX genomic diagnostic test for recurrence prognosis and therapeutic response prediction in node-negative, estrogen receptor-positive breast cancer. Clin Chem 53:1084-1091. doi:10.1373/clinchem.2006.076497

14. Cuzick J, Sestak I, Pinder SE, Ellis IO, Forsyth S, Bundred NJ, Forbes JF, Bishop H, Fentiman IS, George WD (2011) Effect of tamoxifen and radiotherapy in women with locally excised ductal carcinoma in situ: long-term results from the UK/ANZ DCIS trial. Lancet Oncol 12:21-29. doi:10.1016/S1470-2045(10) 70266-7

15. Ernster VL, Ballard-Barbash R, Barlow WE, Zheng Y, Weaver DL, Cutter G, Yankaskas BC, Rosenberg R, Carney PA, Kerlikowske K, Taplin SH, Urban N, Geller BM (2002) Detection of ductal carcinoma in situ in women undergoing screening mammography. J Natl Cancer Inst 94:1546-1554

16. Fisher B, Dignam J, Wolmark N, Mamounas E, Costantino J, Poller W, Fisher ER, Wickerham DL, Deutsch M, Margolese R, Dimitrov N, Kavanah M (1998) Lumpectomy and radiation therapy for the treatment of intraductal breast cancer: findings from National Surgical Adjuvant Breast and Bowel Project B-17. J Clin Oncol 16:441-452

17. Fisher B, Land S, Mamounas E, Dignam J, Fisher ER, Wolmark N (2001) Prevention of invasive breast cancer in women with ductal carcinoma in situ: an update of the National Surgical Adjuvant Breast and Bowel Project experience. Semin Oncol 28:400-418. doi:10.1016/S0093-7754(01)90133-2

18. Fisher ER, Dignam J, Tan-Chiu E, Costantino J, Fisher B, Paik S, Wolmark N (1999) Pathologic findings from the National Surgical Adjuvant Breast Project (NSABP) eight-year update of Protocol B-17: intraductal carcinoma. Cancer 86:429-438

19. Holmberg L, Garmo H, Granstrand B, Ringberg A, Arnesson LG, Sandelin K, Karlsson P, Anderson H, Emdin S (2008) Absolute risk reductions for local recurrence after postoperative radiotherapy after sector resection for ductal carcinoma in situ of the breast. J Clin Oncol 26:1247-1252. doi:10.1200/JCO.2007.12. 7969

20. Hughes LL, Wang M, Page DL, Gray R, Solin LJ, Davidson NE, Lowen MA, Ingle JN, Recht A, Wood WC (2009) Local excision alone without irradiation for ductal carcinoma in situ of the breast: a trial of the Eastern Cooperative Oncology Group. J Clin Oncol 27:5319-5324. doi:10.1200/JCO.2009.21.8560

21. Hughes SJ, Xi L, Gooding WE, Cole DJ, Mitas M, Metcalf J, Bhargava R, Dabbs D, Ching J, Kozma L, McMillan W, Godfrey TE (2009) A quantitative reverse transcription-PCR assay for rapid, automated analysis of breast cancer sentinel lymph nodes. J Mol Diagn 11:576-582. doi:10.2353/jmoldx.2009.090037

22. Jackson LC, Camacho F, Levine EA, Anderson RT, Stewart JH (2008) Patterns of care analysis among women with ductal carcinoma in situ in North Carolina. Am J Surg 195:164-169. doi:10.1016/j.amjsurg.2007.10.001

23. Joslyn SA (2006) Ductal carcinoma in situ: trends in geographic, temporal, and demographic patterns of care and survival. Breast J 12:20-27. doi:10.1111/j.1075-122X.2006.00182.x

24. Kerlikowske K, Molinaro A, Cha I, Ljung BM, Ernster VL, Stewart K, Chew K, Moore DH 2nd, Waldman F (2003) Characteristics associated with recurrence among women with ductal carcinoma in situ treated by lumpectomy. J Natl Cancer Inst 95:1692-1702

25. Kim C, Tang G, Pogue-Geile KL, Costantino JP, Baehner FL, Baker J, Cronin MT, Watson D, Shak S, Bohn OL, Fumagalli D, Taniyama Y, Lee A, Reilly ML, Vogel VG, McCaskill-Stevens W, Ford LG, Geyer CE Jr, Wickerham DL, Wolmark N, Paik S (2011) Estrogen receptor (ESR1) mRNA expression and benefit from tamoxifen in the treatment and prevention of estrogen receptor-positive breast cancer. J Clin Oncol 29:4160-4167. doi: $10.1200 /$ jco.2010.32.9615

26. Lester SC, Bose S, Chen YY, Connolly JL, de Baca ME, Fitzgibbons PL, Hayes DF, Kleer C, O'Malley FP, Page DL, Smith BL, Tan LK, Weaver DL, Winer EP (2009) Protocol for the examination of specimens from patients with invasive carcinoma of the breast. In: CoA (ed) Pathologists. College of American Pathologists, Chicago

27. Lester SC, Bose S, Chen YY, Connolly JL, de Baca ME, Fitzgibbons PL, Hayes DF, Kleer C, O'Malley FP, Page DL, Smith BL, Weaver DL, Winer E (2009) Protocol for the examination of specimens from patients with ductal carcinoma in situ of the breast. Arch Pathol Lab Med 133:15-25. doi:10.1043/ 1543-2165-133.1.15

28. Miller NA, Chapman JA, Fish EB, Link MA, Fishell E, Wright B, Lickley HL, McCready DR, Hanna WM (2001) In situ duct carcinoma of the breast: clinical and histopathologic factors and association with recurrent carcinoma. Breast J 7:292-302

29. Network NCC (2014) NCCN clinical practice guidelines in oncology $\left(\mathrm{NCCN}\right.$ Guidelines $\left.{ }^{\circledR}\right)$ : breast cancer 
30. Paik S, Shak S, Tang G, Kim C, Baker J, Cronin M, Baehner FL, Walker MG, Watson D, Park T, Hiller W, Fisher ER, Wickerham DL, Bryant J, Wolmark N (2004) A multigene assay to predict recurrence of tamoxifen-treated, node-negative breast cancer. N Engl J Med 351:2817-2826. doi:10.1056/NEJMoa041588

31. Rakovitch E, Mihai A, Pignol JP, Hanna W, Kwinter J, Chartier C, Ackerman I, Kim J, Pritchard K, Paszat L (2004) Is expert breast pathology assessment necessary for the management of ductal carcinoma in situ ? Breast Cancer Res Treat 87:265-272. doi:10.1007/s10549-004-9454-8

32. Rakovitch E, Nofech-Mozes S, Narod SA, Hanna W, Thiruchelvam D, Saskin R, Taylor C, Tuck A, Sengupta S, Elavathil L, Jani PA, Done SJ, Miller N, Youngson B, Kong I, Paszat L (2013) Can we select individuals with low risk ductal carcinoma in situ (DCIS)? A population-based outcomes analysis. Breast Cancer Res Treat 138:581-590. doi:10.1007/s10549-0132455-8

33. Rakovitch E, Pignol JP, Chartier C, Hanna W, Kahn H, Wong J, Mai V, Paszat L (2007) The management of ductal carcinoma in situ of the breast: a screened population-based analysis. Breast Cancer Res Treat 101:335-347. doi:10.1007/s10549-006-9302-0

34. Rakovitch E, Pignol JP, Hanna W, Narod S, Spayne J, NofechMozes S, Chartier C, Paszat L (2007) Significance of multifocality in ductal carcinoma in situ: outcomes of women treated with breast-conserving therapy. J Clin Oncol 25:5591-5596. doi:10.1200/JCO.2007.11.4686

35. Schwartz GF, Solin LJ, Olivotto IA, Ernster VL, Pressman PI (2000) Consensus conference on the treatment of in situ ductal carcinoma of the breast, April 22-25, 1999. Cancer 88:946-954

36. Sikand K, Lee AH, Pinder SE, Elston CW, Ellis IO (2005) Sections of the nipple and quadrants in mastectomy specimens for carcinoma are of limited value. J Clin Pathol 58:543-545. doi:10. 1136/jcp.2004.022665

37. Silverstein MJ, Lagios MD, Groshen S, Waisman JR, Lewinsky BS, Martino S, Gamagami P, Colburn WJ (1999) The influence of margin width on local control of ductal carcinoma in situ of the breast. N Engl J Med 340:1455-1461. doi:10.1056/NEJM19990 5133401902
38. Solin LJ (2010) The impact of adding radiation treatment after breast conservation surgery for ductal carcinoma in situ of the breast. J Natl Cancer Inst Monogr 2010:187-192. doi:10.1093/ jncimonographs/lgq020

39. Solin LJ, Gray R, Baehner FL, Butler SM, Hughes LL, Yoshizawa C, Cherbavaz DB, Shak S, Page DL, Sledge GW Jr, Davidson NE, Ingle JN, Perez EA, Wood WC, Sparano JA, Badve S (2013) A multigene expression assay to predict local recurrence risk for ductal carcinoma in situ of the breast. J Natl Cancer Inst 105:701-710. doi:10.1093/jnci/djt067

40. Staradub VL, Messenger KA, Hao N, Wiley EL, Morrow M (2002) Changes in breast cancer therapy because of pathology second opinions. Ann Surg Oncol 9:982-987

41. Therneau TM, Grambsch PM (2000) Modeling survival data: extending the cox model. Statistics for biology and health. Springer, New York

42. Wapnir IL, Dignam JJ, Fisher B, Mamounas EP, Anderson SJ, Julian TB, Land SR, Margolese RG, Swain SM, Costantino JP, Wolmark N (2011) Long-term outcomes of invasive ipsilateral breast tumor recurrences after lumpectomy in NSABP B-17 and B-24 randomized clinical trials for DCIS. J Natl Cancer Inst 103:478-488. doi:10.1093/jnci/djr027

43. Warren JL, Weaver DL, Bocklage T, Key CR, Platz CE, Cronin KA, Ballard-Barbash R, Willey SC, Harlan LC (2005) The frequency of ipsilateral second tumors after breast-conserving surgery for DCIS: a population based analysis. Cancer 104:1840 1848. doi:10.1002/cner.21406

44. Westfall PH, Krishen A (2001) Optimally weighted, fixed sequence and gatekeeper multiple testing procedures. J Stat Plan Inference 99:25-40

45. Wong JS, Kaelin CM, Troyan SL, Gadd MA, Gelman R, Lester SC, Schnitt SJ, Sgroi DC, Silver BJ, Harris JR, Smith BL (2006) Prospective study of wide excision alone for ductal carcinoma in situ of the breast. J Clin Oncol 24:1031-1036. doi:10.1200/ JCO.2005.02.9975

46. Zujewski JA, Harlan LC, Morrell DM, Stevens JL (2011) Ductal carcinoma in situ: trends in treatment over time in the US. Breast Cancer Res Treat 127:251-257. doi:10.1007/s10549-010-1198-Z 